\title{
Examination of Efficiency Based on Air Gap and Characteristic Impedance Variations for Magnetic Resonance Coupling Wireless Energy Transfer
}

\author{
Ali Agcal, Nur Bekiroglu, and Selin Ozcira* \\ Electrical Engineering Department, Yildiz Technical University, Davutpasa, 34220, Istanbul, Turkey
}

(Received 1 September 2014, Received in final form 13 January 2015, Accepted 13 January 2015)

\begin{abstract}
In this paper wireless power transmission system based on magnetic resonance coupling circuit was carried out. With the research objectives based on the mutual coupling model, mathematical expressions of optimal coupling coefficients are examined. Equivalent circuit parameters are calculated by Maxwell software, and the equivalent circuit was solved by Matlab software. The power transfer efficiency of the system was derived by using the electrical parameters of the equivalent circuit. System efficiency was analyzed depending on the different air gap values for various characteristic impedances. Hence, magnetic resonance coupling involves creating a resonance and transferring the power without radiating electromagnetic waves. As the air gap between the coils increased the coupling between the coils were weakened. The impedance of circuit varied as the air gap changed, affecting the power transfer efficiency.
\end{abstract}

Keywords : wireless power transfer, magnetic coupling, characteristic impedance, efficiency

\section{Introduction}

Recently, a wireless power transfer in the medium range has been comprehensively researched for its applications to consumer electronics, portable devices, robotics, and electrical vehicles. General research activities on the noncontact power transfer systems in the EV system also have increased during the recent years, due to the problems associated with short battery life and traditional chargers which must be physically connected to EV. Wireless power transfer technology can electrically conduct energy from a source to electric device without interconnecting any medium [1]. WPT via strongly coupled magnetic resonances in the near field has been reported [2,3]. The basic principle of WPT, based on magnetically coupled resonance, is that two self-resonators with the same resonant frequency can efficiently transfer energy over the midrange distances [4]. Although the magnetic-coupled resonant WPT technology is the epoch-making technology, it seems to be similar to magnetic induction. Both of them use magnetic field to transfer power and magnetic coil to feed/pickup power [5]. There have been so many efforts involved, such as modification circuits and magnetic

(C)The Korean Magnetics Society. All rights reserved.

*Corresponding author: Tel: +90-505-322-4805

Fax:+90-212-383-5858, e-mail: selinozcira@gmail.com design of core properties $[6,7]$. Witricity is a new wireless powering technique which was reported by a Massachusetts Institute of Technology (MIT) research team in 2007. It is able to wirelessly transmit power over a long distance, in the order of several times of the physical size of the transmitter/receiver, based on strongly coupled magnetic resonance [8]. Instead of spreading radiative and lossy electromagnetic fields to the environment, the source resonator fills the surrounding with lossless non-radiative magnetic fields oscillating at megahertz frequencies. The non-radiative fields allow efficient power exchange between the source and the device resonators. There are two major disadvantages, regarding physical sizes, with magnetic coupling in wireless power transfer technology. If the size of the receiver is reduced, the magnetic flux linked to the secondary coil is greatly reduced. In order to ensure that the magnetic flux is sufficiently high for the efficient energy transfer, a large current must flow through the primary coil [9]. As an essential approach, an evaluation is needed for the energy transmission efficiency. Thus, system efficiency should be analyzed for various characteristic impedances, depending on different air gap values. In this study, efficiency has been examined and shown with respect to the characteristic impedance. Magnetic resonant coupling system through equivalent circuit simulations is demonstrated. Equivalent circuit solved in Matlab platform and a procedure to calculate parameters of the equivalent 
circuit had been carried out in Maxwell.

\section{Efficiency Calculation by Magnetic Resonant Coupling Equivalent Circuit}

In this section, equivalent circuit is explained, which is a basic theory of this paper. To derive the analytical equations of the input impedance and transferred power, a simplified equivalent circuit model of the WPT systems is presented in Fig. 1.

Each loop represents the electromotive force induced in each coil by the current flowing in the opposite coil, and are related by the impedance of the mutual inductance at resonance.

The magnetic coupling $(k$ is the magnetic coupling factor, $0<k<1$ ) is formed between transmitter coil $L_{1}$ and receiver coil $L_{2} . M$ is the mutual inductance. The load impedance is calculated based on the simplified equivalent circuit model. The impedance $Z_{\text {load }}=Z_{0}$ is given by,

$$
Z_{\text {load }}=Z_{0}=\frac{\left(\left(j \omega C_{1}\right) R_{s}+1-\omega^{2} L_{1} C_{1}\right)-\left(\left(j \omega C_{2}\right) R_{2}+1-\omega^{2} L_{2} C_{2}\right)}{j \omega C_{1}\left(\left(j \omega C_{2}\right) R_{2}+1-\omega^{2} L_{2} C_{2}\right)}
$$

To simplify the calculations of the resonant frequencies, $\mathrm{R}_{1}$ and $\mathrm{R}_{2}$ are ignored. The circuit shown in Fig. 1 is a model of a wireless power system created by using a pair of magnetically coupled resonators.

For a parallel resonance, the input impedance would be infinity. But for series resonances, the input impedance should be zero. Eq. (1) should be rearrange as,

$$
Z_{\text {load }}=Z_{0}=\frac{\sqrt{L_{1} C_{1}+L_{2} C_{2}+\sqrt{L_{1}^{2} C_{1}^{2}+L_{2}^{2} C_{2}^{2}-2\left(1-2 k^{2}\right) L_{1} C_{1} L_{2} C_{2}}}}{2 \pi \sqrt{2\left(1-2 k^{2}\right) L_{1} C_{1} L_{2} C_{2}}}
$$

The power delivered to the characteristic impedance $Z_{0}$ is maximized, when the impedances of the inductors $L_{1}$

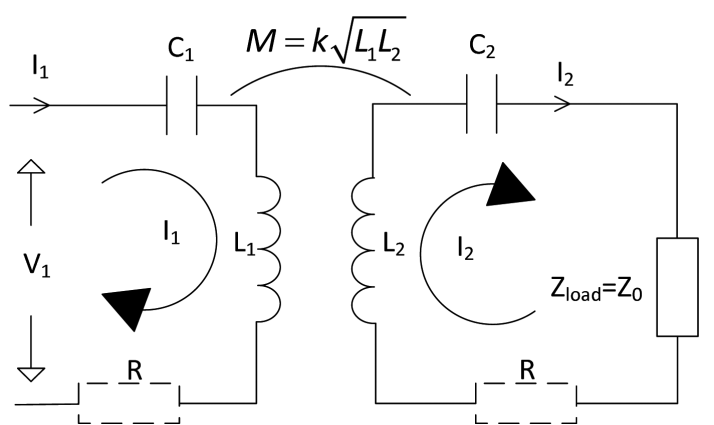

Fig. 1. Simplified equivalent circuit model of wireless power transfer systems.

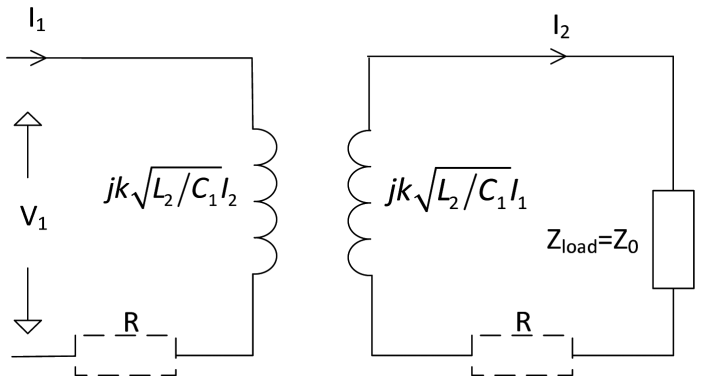

Fig. 2. Equivalent circuit for magnetic resonant coupling.

and $L_{2}$ are canceled by the impedance of the capacitors $C_{1}$ and $C_{2}$. Assuming that the system is designed such that these two resonant frequencies coincide, the circuit model reduces to the one shown in Fig. 2.

Although a way of detecting efficiency of transmission in an electromagnetic network is to look at the scattering parameters, the major aim in this study is to determine the efficiency by using electrical correlations. Assuming $C_{1}=$ $C_{2}$ in the resonance coupling system, the efficiency can be defined by Eq. (3)

$$
\begin{aligned}
& \eta=\frac{P_{2}}{P_{1}}=\frac{I_{2}^{2} Z_{2}}{I_{1}^{2} Z_{2}} \\
& I_{2}=I_{1} \frac{j M \omega}{j L_{2} \omega+\left(\frac{1}{j \omega C}\right)+Z_{0}+R}
\end{aligned}
$$

By substituting Eq. (2) and Eq. (4) in Eq. (3), efficiency can be obtained by Eq. (5).

$$
\begin{aligned}
& \eta=\left(\frac{j \omega C}{j L_{2} \omega+\left(\frac{1}{j \omega C}\right)+Z_{0}+R}\right) \\
& \left(R+j L_{1} \omega+\left(\frac{1}{j \omega C}\right)+\left(\frac{Z_{0}}{j L_{2} \omega+\left(\frac{1}{j \omega C}\right)+Z_{0}+R}\right)\right)
\end{aligned}
$$

Based on the reference [10], the emitting and receiving coils have four different resonance coupling states as follows: first condition, both the emitting and receiving coil loop are not resonant; second condition, the emitting coil loop is in resonance, while the receiving coil loop is not resonant; third condition, the emitting coil loop is not resonant, while receiving coil is in resonance; and the last condition, both the emitting and receiving coil loop are resonant. When the emitting and receiving coil are resonant, the whole coupling system is in resonance; the resonant coil circuit is pure resistance loop and the impedance is at 
minimum; and the current flowing through the resonant coil is the highest, when the power and efficiency are at best, compared to the other three states.

\section{Parameters calculated by MoM and the equivalent circuit model}

Under the condition which is defined in Eq. (6), the system has double resonance frequency; and for Eq. (7), the system has one resonance frequency that provides low efficiency [11].

$$
\begin{aligned}
& M^{2}>\frac{Z_{0}^{2}-R^{2}}{\omega_{0}^{2}} \\
& M^{2}<\frac{Z_{0}^{2}-R^{2}}{\omega_{0}^{2}}
\end{aligned}
$$

While performing the simulations both situations have been encountered. Equivalent circuit parameters were calculated by Maxwell software with the respect to the method of moments, and the equivalent circuit was solved by Matlab software. Efficiency can be determined according to load variation for different air gap values. The parameters of the system reproducing parameter $L$ is a nonlinear inductance dependent on current. The parameter $M$ is a nonlinear mutual inductance, which is dependent on the current and frequency. Each parameter was calculated independently. Resonance frequency is a key parameter in the system design, whose value can be changed by adjusting distance between the transmission and characteristic impedance of the electrical circuit. In summary, by appropriate readjustment of the system parameters, frequency can be acquired for various operating states. These operating parameters are given in Table 1, and the

Table 1. Circuit parameters calculated by Maxwell to use simulation of magnetic resonant coupling circuit.

\begin{tabular}{cccccc}
\hline \hline Parameter/Air gap & $1 \mathrm{~cm}$ & $5 \mathrm{~cm}$ & $10 \mathrm{~cm}$ & $15 \mathrm{~cm}$ & $20 \mathrm{~cm}$ \\
\hline $\mathrm{L}[\mathrm{nH}]$ & 1004 & 982.4 & 999.2 & 1010.5 & 996.7 \\
$\mathrm{M}[\mathrm{nH}]$ & 562.5 & 241.8 & 128.6 & 80.5 & 46.2 \\
$\mathrm{C}[\mathrm{pF}]$ & 124 & 124 & 124 & 124 & 124 \\
\hline
\end{tabular}

Table 2. The resonance frequency values in $\mathrm{MHz}$ according to air gap and the characteristic impedance variation.

\begin{tabular}{ccccccc}
\hline \hline Air gap $/ Z_{0}$ & $1 \Omega$ & $5 \Omega$ & $10 \Omega$ & $25 \Omega$ & $50 \Omega$ & $100 \Omega$ \\
\hline $1 \mathrm{~cm}$ & 11.43 & 11.45 & 11.49 & 11.87 & 14.21 & 18.15 \\
$5 \mathrm{~cm}$ & 12.92 & 12.96 & 13.10 & 14.68 & 15.14 & 17.30 \\
$10 \mathrm{~cm}$ & 13.46 & 13.56 & 13.92 & 14.47 & 14.91 & 16.94 \\
$15 \mathrm{~cm}$ & 13.69 & 13.85 & 14.25 & 14.37 & 14.80 & 16.78 \\
$20 \mathrm{~cm}$ & 14.01 & 14.32 & 14.34 & 14.46 & 14.90 & 16.92 \\
\hline
\end{tabular}

frequency values according to air gap and the characteristic impedance variation are given in Table 2.

As stated above, maximum efficiency occurs at the resonance frequency. As the air gap between coils was increased, the coupling between the coils was weakened. The impedance of the circuit will change as the air gap changes, which affects the power transfer efficiency.

\section{Results and Discussion}

By using equivalent circuit, the frequency characteristics and power transfer efficiency of the coils have been calculated, and electromagnetic analysis simulations have been carried out. The results showed that the equivalent circuit model can be used to estimate the frequency characteristics.

\subsection{Frequency characteristics}

Considering all of the states for the contents of Table 1

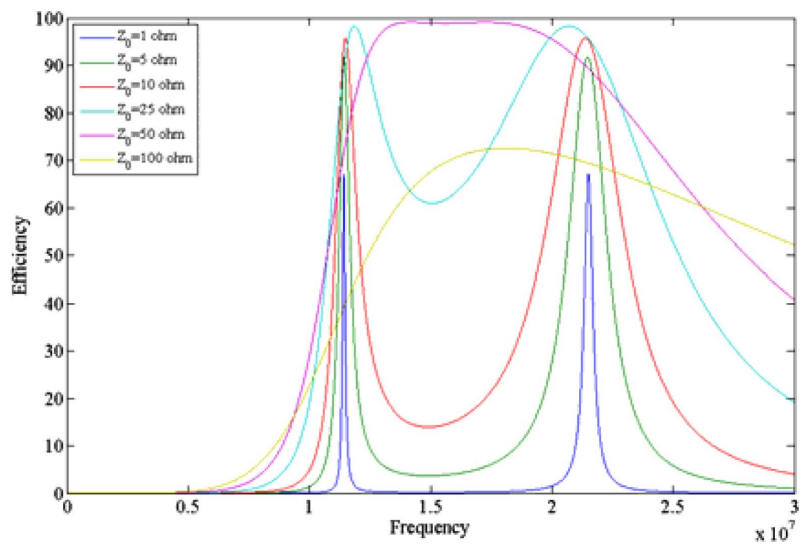

Fig. 3. (Color online) Air gap $=1 \mathrm{~cm}$, efficiency vs frequency graphics for $Z_{0}=1 \Omega, Z_{0}=5 \Omega, Z_{0}=10 \Omega, Z_{0}=25 \Omega, Z_{0}=50$ $\Omega, Z_{0}=100 \Omega$.

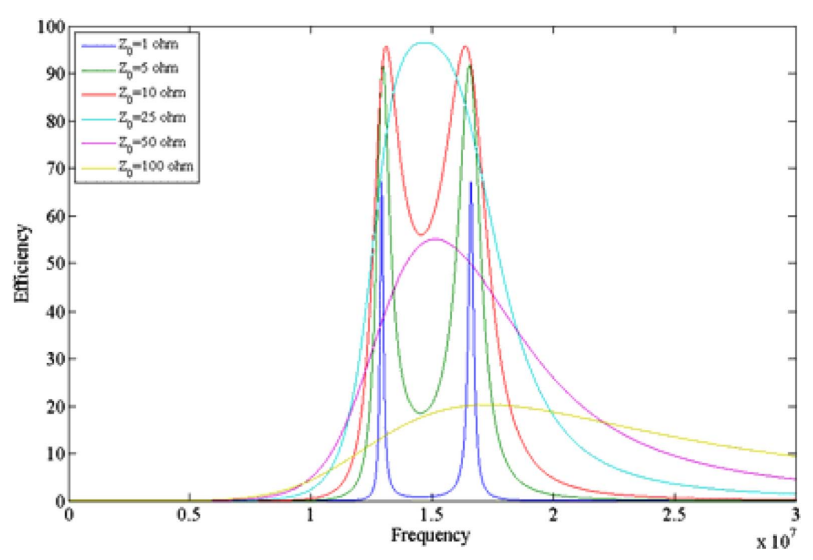

Fig. 4. (Color online) Air gap $=5 \mathrm{~cm}$, efficiency vs frequency graphics for $Z_{0}=1 \Omega, Z_{0}=5 \Omega, Z_{0}=10 \Omega, Z_{0}=25 \Omega, Z_{0}=50$ $\Omega, Z_{0}=100 \Omega$. 


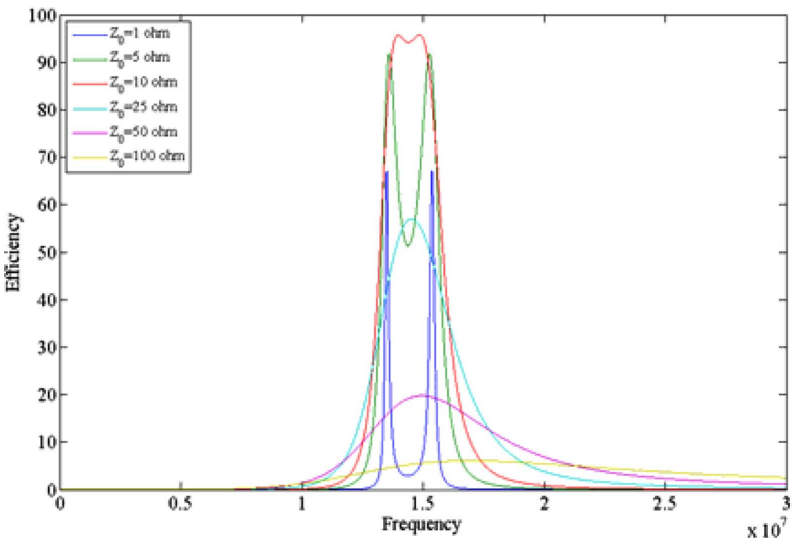

Fig. 5. (Color online) Air gap $=10 \mathrm{~cm}$, efficiency vs frequency graphics for $Z_{0}=1 \Omega, Z_{0}=5 \Omega, Z_{0}=10 \Omega, Z_{0}=25 \Omega$, $Z_{0}=50 \Omega, Z_{0}=100 \Omega$.

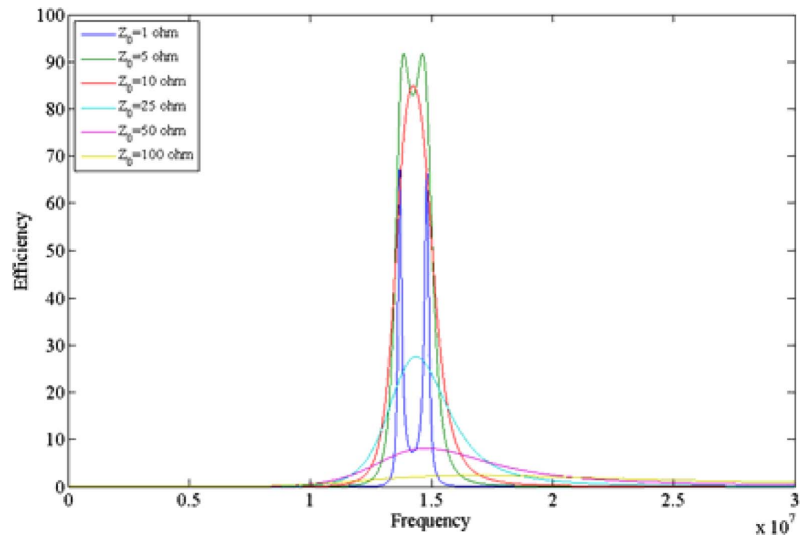

Fig. 6. (Color online) Air gap $=15 \mathrm{~cm}$, efficiency vs frequency graphics for $Z_{0}=1 \Omega, Z_{0}=5 \Omega, Z_{0}=10 \Omega, Z_{0}=25 \Omega$, $Z_{0}=50 \Omega, Z_{0}=100 \Omega$.

and Table 2, Eq. (5) was solved in respect to the magnetic resonant coupling equivalent circuit parameters. Efficiency vs frequency graphs for each state are given below.

Efficiency with respect to load variation (characteristic impedance) results showed that there are double resonance frequency regions as well as one resonance region; and the resonance frequency changed when the air gap was changed. The resonant frequencies changed from two points to one point, depending on the length of the air gap. But the maximum efficiency at resonant frequency was not changed until a certain distance. The efficiencies were approximately 95\%, with the certain convenient distance as $5 \mathrm{~cm}-10 \mathrm{~cm}$.

\subsection{Findings on the idealized system based on theo- retical approach}

The findings presented above are based on calculations made by an approach with idealized theory. Since the

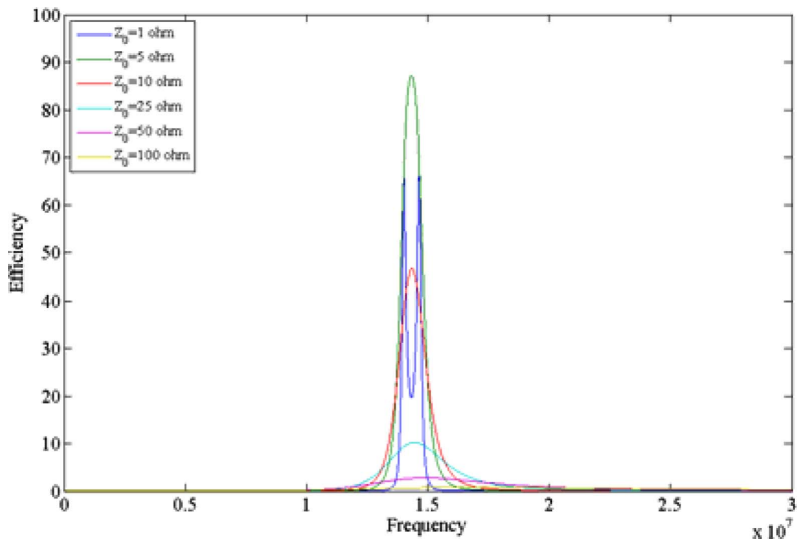

Fig. 7. (Color online) Air gap $=20 \mathrm{~cm}$, efficiency vs frequency graphics for $Z_{0}=1 \Omega, Z_{0}=5 \Omega, Z_{0}=10 \Omega, Z_{0}=25 \Omega$, $Z_{0}=50 \Omega, Z_{0}=100 \Omega$.

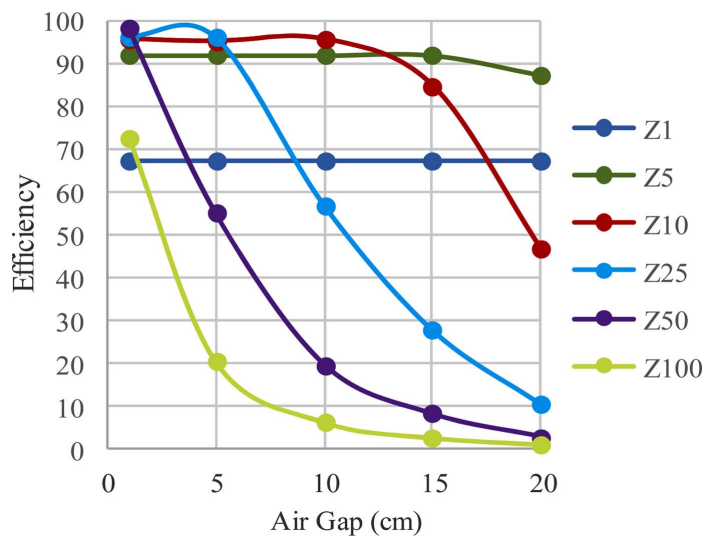

Fig. 8. (Color online) According to different characteristic impedances efficiency-air gap chart.

characteristic impedance is a critical parameter for the maximum efficiency of the wireless power transfer, this research emphasizes on matching the load variation $\left(Z_{0}\right)$. The efficiency was decreased when the distance increased from $10 \mathrm{~cm}-20 \mathrm{~cm}$ for $Z_{0}=10 \Omega$. The system offers a

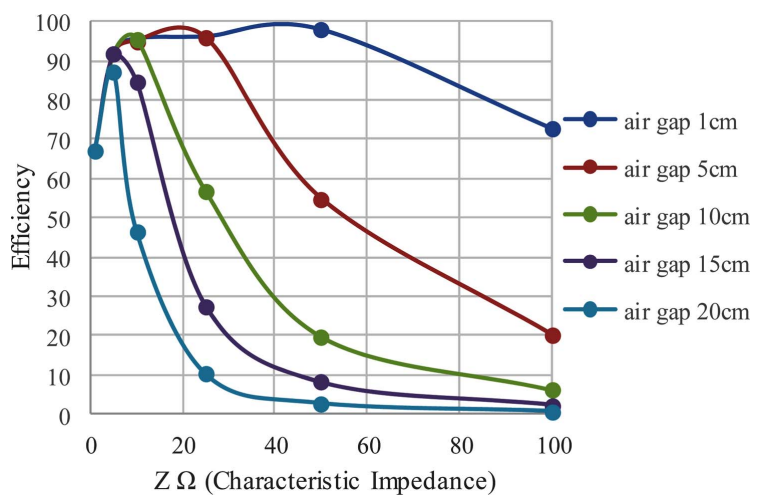

Fig. 9. (Color online) Characteristic impedance chart according to different air gap values efficiency. 
much more efficient power transfer at that point. Efficiency of $Z_{0}=50 \Omega$ and air gap $=1 \mathrm{~cm}$ configuration is the highest one.

As shown in Fig. 8, while the characteristic impedance increases, the system operates at high mutual inductance and closest distance in order to achieve higher efficiency.

As shown in Fig. 9, the system operates on one resonance frequency by the means of increasing the distance and the characteristic impedance; and when the air gap and the characteristic impedance further increases, the efficiency yields drop sharply.

\section{Conclusions}

Recently, wireless power transfer over a medium range via magnetic resonance coupling is being extensively researched for various applications. In this study, wireless power transfer has been analyzed by employing the transient analysis, and the equivalent circuit was solved in order to achieve the efficiency value.

The numerical results based on various air gap values were determined, in the view of equivalent circuit parameters. Equivalent circuit parameters were calculated from the values directly obtained from Maxwell, from the results of electromagnetics analysis. The calculation procedure of mutual inductance between two self-resonators was explained and performed by Maxwell software, as well. Equivalent circuit was solved in Matlab platform. Mom calculation showed that when using the parameters which are obtained by mom analysis, the equivalent circuit can hold a capability to calculate the efficiency by utilizing the equivalent circuit to design a matching network.

\section{References}

[1] P. Kotchapansompote, W. Yafei, T. Imura, H. Fujimoto, and Y. Hon, 37th Annual Conference on IEEE Industrial Electronics Society (IECON), 1 (2011).

[2] A. Bodrov and S. K. Sul, Wireless Power Transfer-Principles and Engineering Explorations, InTech, Croatia (2012) pp. 9-50.

[3] T. Imura, H. Okabe, and Y. Hori, Vehicle Power and Propulsion Conference (VPPC), 1 (2009).

[4] Y. Park, J. Kim, and K. H. Kim, Wireless Power Transfer-Principles and Engineering Explorations, InTech, Croatia (2012) pp. 51-64.

[5] H. Hirayama, Wireless Power Transfer-Principles and Engineering Explorations, InTech, Croatia (2012) pp. 117-132.

[6] J. H. Park and S. W. Kim, J. Magn. 18, 105 (2013).

[7] R. A. Salasa and J. Pleite, J. Appl. Phys. 107, 09A517 (2010).

[8] A. Kurs, A. Karalis, R. Mofatt, J. D. Joannopoulos, P. Fisher, and M. Soljacic, Science 317, 83 (2007).

[9] J. Wang, S. L. Ho, W. Fu, C. T. Kit, and M. Sun, IEEE Trans. Magn. 47, 1074 (2011).

[10] Q. Wang and H. Li, International Conference Electronics, Communications and Control (ICECC), 1 (2011).

[11] T. Imura and Y. Hori, IEEE Trans. Ind. Elect. 58, 4746 (2011).

[12] K. E. Koh, T. C. Beh, T. Imura, and Y. Hori, IEEE Trans. Ind. Appl. 50, 2061 (2014). 